\title{
Variable-length Feedback Codes with Several Decoding Times for the Gaussian Channel
}

\author{
Recep Can Yavas, Victoria Kostina, and Michelle Effros
}

\begin{abstract}
We investigate variable-length feedback (VLF) codes for the Gaussian point-to-point channel under maximal power, average error probability, and average decoding time constraints. Our proposed strategy chooses $K<\infty$ decoding times $n_{1}, n_{2}, \ldots, n_{K}$ rather than allowing decoding at any time $n=0,1,2, \ldots$ We consider stop-feedback, which is one-bit feedback transmitted from the receiver to the transmitter at times $n_{1}, n_{2}, \ldots$ only to inform her whether to stop. We prove an achievability bound for VLF codes with the asymptotic approximation $\ln M \approx \frac{N C(P)}{1-\epsilon}-\sqrt{N \ln _{(K-1)}(N) \frac{V(P)}{1-\epsilon}}$, where $\ln _{(K)}(\cdot)$ denotes the $K$-fold nested logarithm function, $N$ is the average decoding time, and $C(P)$ and $V(P)$ are the capacity and dispersion of the Gaussian channel, respectively. Our achievability bound evaluates a non-asymptotic bound and optimizes the decoding times $n_{1}, \ldots, n_{K}$ within our code architecture.
\end{abstract}

Index Terms-Variable-length stop-feedback codes, Gaussian channel, second-order achievability bound.

\section{INTRODUCTION}

Although Shannon's work [1] shows that feedback does not increase the capacity of memoryless, point-to-point channels, it is known that feedback has several important benefits in channel coding such as simplifying coding schemes and improving higher-order achievable rates. Several results demonstrate this effect in the fixed-length regime. Feedback simplifies coding in Horstein's scheme for the binary symmetric channel [2] and Schalkwijk and Kailath's scheme [3] for the Gaussian channel. Wagner et al. [4] show that feedback improves the second-order achievable rate for any discrete memoryless channel (DMC) with multiple capacity-achieving input distributions giving distinct dispersions.

The benefits of feedback increase for codes with arbitrary decoding times (called variable-length or rateless codes). In [5], Burnashev shows that feedback significantly improves the optimal error exponent of variable-length codes for DMCs. In [6], Polyanskiy et al. extend the work of Burnashev to the finite-length regime with non-vanishing error probabilities, introducing variable-length feedback (VLF) and variablelength feedback with termination (VLFT) codes, and deriving achievability and converse bounds for their performance. In VLF codes, the receiver decides when to stop transmissions and decode; in VLFT codes, the transmitter decides when to stop transmissions using its knowledge of the source message and the feedback that it receives from the receiver. As a

R. C. Yavas, V. Kostina, and M. Effros are with the Department of Electrical Engineering, California Institute of Technology, Pasadena, CA 91125, USA (e-mail: ryavas, vkostina, effros@caltech.edu). This work was supported in part by the National Science Foundation (NSF) under grant CCF-1817241 and CCF-1956386. special case of VLF codes, Polyanskiy et al. define variablelength stop-feedback (VLSF) codes that use feedback from the receiver to the transmitter at each potential decoding time to inform the transmitter whether to stop transmitting; in this regime, the codewords are fixed when the code is designed, and feedback affects how much of a codeword is sent but not that codeword's value. The result in [6, Th. 2] shows that variable-length decoding improves the first-order term in the asymptotic expansion of the maximum achievable message size from $N C$ to $\frac{N C}{1-\epsilon}$, where $C$ is the capacity of the DMC, $N$ is the average decoding time, and $\epsilon$ is the average error probability. The second-order achievable term within the class of VLF and VLFT codes is $O(\ln N)$, which means that VLF and VLFT codes have zero dispersion, and the convergence to the capacity is much faster than that achieved by the fixedlength codes [7], [8].

Variations of VLSF and VLFT codes are studied in [9][15]. In [9], Kim et al. consider VLSF codes where decoding must occur at a decoding time less than or equal to some constant $\ell$ and the decoding times satisfy $n_{i}=i d$ for some $d \in \mathbb{Z}^{+}$. In [10], Altuğ et al. modify the VLSF coding paradigm by replacing the average decoding time constraint with a constraint on the probability that the decoding time exceeds a target value; the benefit in the first-order term does not appear under this probabilistic delay constraint [10]. Truong and Tan [11], [12] extend the results in [6] to the Gaussian point-to-point and multiple access channels under an average power constraint. Trillingsgaard et al. [13] study the VLSF scenario where a common message is transmitted across a $K$-user discrete memoryless broadcast channel.

For the Gaussian channel, the effect of feedback depends both on whether the power constraint limits maximum or average power ${ }^{1}$ and on whether the code is fixed-length or variable-length. For example, in the fixed-length regime, feedback does not improve the code performance in its first-, second-, or third-order terms under the maximal power constraint [8], but does improve the achievable second-order term under the average power constraint [16]. In the variablelength regime where the decoder can decode at any time and an average power constraint is employed, feedback improves performance in the first-order term [11], [12].

While high rates of feedback are impractical for many applications - especially wireless applications on half-duplex devices most prior work on VLSF codes [9]-[13] allows an unbounded number of potential decoding times, i.e., $K=\infty$.

\footnotetext{
${ }^{1}$ The maximal power constraint is also called the short-term, per-codeword, or peak power constraint; the average power constraint is also known as the long-term or expected power constraint.
} 
Exceptions include [14], which numerically evaluates the performance of a VLSF code with $K<\infty$ decoding times using a new reliability-output Viterbi algorithm at the decoder, and [15], which introduces a sequential differential optimization algorithm to optimize the choices of $K$ potential decoding times $n_{1}, \ldots, n_{K}$, approximating the probability $\mathbb{P}[\tau \leq n]$ that random stopping time $\tau$ is no greater than $n$ by a differentiable function $F(n)$.

This paper presents the first asymptotic expansion for the rate achievable by VLSF codes between the two extremes: $K=1$ (the fixed-length regime analyzed in [7], [17]), and $K=\infty$ (the classical variable-length regime defined in [6, Def. 1]). We consider VLSF codes over the Gaussian pointto-point channel and limit the number of decoding times to some finite integer $K$ that does not grow with the average decoding time $N$. We impose a new maximal power constraint, bounding the power of codewords at every potential decoding time. The feedback rate of our code is $\frac{k}{n_{k}}$ when the decoding time is $n_{k}$. Thus our feedback rate approaches 0 as $n_{k}$ grows for each $n_{k}$ while most other VLSF codes use feedback rate 1 bit per channel use. Throughout the paper, we employ the average error and average decoding time criteria. Our main result shows that for VLSF codes with $2 \leq K<\infty$ decoding times, message set size $M$ satisfying

$$
\ln M \approx \frac{N C(P)}{1-\epsilon}-\sqrt{N \ln _{(K-1)}(N) \frac{V(P)}{1-\epsilon}}
$$

is achievable. Here $\ln _{(K)}(\cdot)$ denotes the $K$-fold nested logarithm function, $N$ is the average decoding time, and $C(P)$ and $V(P)$ are the capacity and dispersion of the Gaussian channel, respectively. The order of the second-order term in (1) depends on $K$. The convergence to $\frac{C(P)}{1-\epsilon}$ in (1) is slower than the convergence to $C(P)$ in the fixed-length scenario, which has second-order term $O(\sqrt{N})$ [7]. The $K=2$ case in (1) recovers the variable-length scenario without feedback, which has second-order term $O(\sqrt{N \ln N})$ [6, Th. 1], achieved with $n_{1}=0$; our bound in Theorem 4 shows that when $K=\infty$, the asymptotic approximation in (1) is achievable with the second-order term replaced by $-O(\sqrt{N})$. Our result in (1) demonstrates how the performance of VLSF codes interpolates between these two extremes. Despite the orderwise dependence on $K$, (1) grows so slowly with $K$ that it suggests little benefit to choosing a large $K$. For example, when $K=4, \sqrt{N \ln _{(K-1)}(N)}$ behaves very similarly to $O(\sqrt{N})$ for practical values of $N$ (e.g., $N \in\left[10^{3}, 10^{5}\right]$ ). Notice, however that the given achievability result provides a lower bound on the benefit of increasing $K$; bounding the benefit from above would require a converse, a topic left to future work. Table I, below, combines the $K=1$ summary from [16, Table I] with the corresponding results for $K>1$ to summarize the performance of VLSF codes for the Gaussian channel in different communication scenarios.

In what follows, Section II introduces VLSF codes with $K$ decoding times, and Section III presents our main results and discusses their implications. Proofs are found in Section IV.

\section{Problem Statement}

\section{A. Notation}

For any positive integers $k$ and $n,[k] \triangleq\{1, \ldots, k\}$ and $x^{n} \triangleq\left(x_{1}, \ldots, x_{n}\right)$. For any $x^{n}$, and integers $n_{1} \leq n_{2} \leq n$, $x_{n_{1}}^{n_{2}} \triangleq\left(x_{n_{1}}, x_{n_{1}+1}, \ldots, x_{n_{2}}\right)$. All-zero and all-one vectors are denoted by $\mathbf{0}$ and $\mathbf{1}$, respectively. The identity matrix of dimension $n$ is denoted by $\mathrm{I}_{n}$. We denote the minimum of two scalars $a$ and $b$ by $a \wedge b$. The Euclidean norm of vector $x^{n}$ is denoted by $\left\|x^{n}\right\| \triangleq \sqrt{\sum_{i=1}^{n} x_{i}^{2}}$. The $n$-dimensional sphere with radius $r$ is denoted by $\mathbb{S}^{n}(r) \triangleq\left\{x^{n} \in \mathbb{R}^{n}:\left\|x^{n}\right\|=r\right\}$. We use $\ln (\cdot)$ to denote the natural logarithm. We measure information in nats. We use the standard $O(\cdot)$ and $o(\cdot)$ notations, i.e., $f(n)=O(g(n))$ if $\limsup _{n \rightarrow \infty}|f(n) / g(n)|<\infty$ and $f(n)=o(g(n))$ if $\lim _{n \rightarrow \infty}|f(n) / g(n)|=0$. We denote the distribution of a random variable $X$ by $P_{X}$, and we write $\mathcal{N}(\boldsymbol{\mu}, \mathrm{V})$ to denote the multivariate Gaussian distribution with mean $\boldsymbol{\mu}$ and covariance matrix $\mathrm{V}$. We use $Q(\cdot)$ to represent the complementary Gaussian cumulative distribution function $Q(x) \triangleq \frac{1}{\sqrt{2 \pi}} \int_{x}^{\infty} \exp \left\{-\frac{t^{2}}{2}\right\} d t$ and $Q^{-1}(\cdot)$ to represent its functional inverse.

The $k$-fold nested logarithm function is defined as

$\ln _{(k)}(x) \triangleq \begin{cases}\ln (x) & \text { if } k=1, x>0 \\ \ln \left(\ln _{(k-1)}(x)\right) & \text { if } k>1, \ln _{(k-1)}(x)>0\end{cases}$

and undefined for all other $(k, x)$ pairs.

\section{B. Channel Model}

The output of a memoryless, point-to-point Gaussian channel in response to input $X^{n} \in \mathbb{R}^{n}$ is

$$
Y^{n}=X^{n}+Z^{n}
$$

where $Z_{1}, \ldots, Z_{n}$ are $\mathcal{N}(0,1)$ random variables independent of $X^{n}$ and of each other.

The channel's capacity and dispersion are

$$
\begin{aligned}
& C(P) \triangleq \frac{1}{2} \ln (1+P) \\
& V(P) \triangleq \frac{P(P+2)}{2(1+P)^{2}},
\end{aligned}
$$

respectively. The information density of a channel $P_{Y^{n} \mid X^{n}}$ under input distribution $P_{X^{n}}$ is defined as

$$
\imath\left(x^{n} ; y^{n}\right) \triangleq \ln \frac{P_{Y^{n} \mid X^{n}}\left(y^{n} \mid x^{n}\right)}{P_{Y^{n}}\left(y^{n}\right)},
$$

where $P_{Y^{n}}$ is the marginal of $P_{X^{n}} P_{Y^{n} \mid X^{n}}$.

\section{VLSF Codes with K Decoding Times}

We consider VLSF codes with a finite number of potential decoding times $n_{1}<n_{2}<\cdots<n_{K}$. The receiver chooses to end the transmission at the first time $n_{k} \in\left\{n_{1}, \ldots, n_{K}\right\}$ that it is ready to decode. The transmitter learns of the receiver's decision via a single bit of feedback at each of times $n_{1}, \ldots, n_{k}$. Feedback bit " 0 " at time $n_{i}$ means that the receiver is not yet ready to decode and the transmitter should continue; feedback bit " 1 " means that the receiver can decode at time $n_{i}$ and the transmitter must stop. We 
TABLE I

THE PERFORMANCE OF VLSF CODES FOR THE GAUSSIAN CHANNEL IN SCENARIOS DISTINGUISHED BY THE NUMBER OF AVAILABLE DECODING TIMES $K$, THE TYPE OF THE POWER CONSTRAINT, AND THE PRESENCE OF FEEDBACK.

\begin{tabular}{|c|c|c|c|c|c|}
\hline & & & \multirow{2}{*}{ First-order term } & \multicolumn{2}{|c|}{ Second-order term } \\
\hline & & & & Lower Bound & Upper Bound \\
\hline \multirow{4}{*}{$\begin{array}{l}\text { Fixed-length } \\
\qquad(K=1)\end{array}$} & \multirow{2}{*}{ No Feedback } & Max. power & $N C(P)$ & $-\sqrt{N V(P)} Q^{-1}(\epsilon) \quad([7],[17])$ & $-\sqrt{N V(P)} Q^{-1}(\epsilon)$ \\
\hline & & Ave. power & $N C\left(\frac{P}{1-\epsilon}\right)$ & $-\sqrt{N \ln N V\left(\frac{P}{1-\epsilon}\right)}$ & $-\sqrt{N \ln N V\left(\frac{P}{1-\epsilon}\right)} \quad([18])$ \\
\hline & \multirow{2}{*}{ Feedback } & Max. power & $N C(P)$ & $-\sqrt{N V(P)} Q^{-1}(\epsilon) \quad$ [7], [17] & $-\sqrt{N V(P)} Q^{-1}(\epsilon)$ \\
\hline & & Ave. power & $N C\left(\frac{P}{1-\epsilon}\right)$ & $-O\left(\ln _{(K)}(N)\right)$ & $+\sqrt{N \ln N V\left(\frac{P}{1-\epsilon}\right)} \quad([16])$ \\
\hline \multirow{2}{*}{$\begin{array}{l}\text { Variable-length } \\
\qquad(K<\infty)\end{array}$} & \multicolumn{2}{|c|}{ Max. power } & $\frac{N C(P)}{1-\epsilon}$ & $-\sqrt{N \ln _{(K-1)}(N) \frac{V(P)}{1-\epsilon}} \quad($ Theorem 1$)$ & $+O(1) \quad([11])$ \\
\hline & \multicolumn{2}{|c|}{ Ave. power } & $\frac{N C(P)}{1-\epsilon}$ & $-\sqrt{N \ln _{(K-1)}(N) \frac{V(P)}{1-\epsilon}} \quad($ Theorem 1$)$ & $+O(1) \quad([11])$ \\
\hline \multirow{2}{*}{$\begin{array}{l}\text { Variable-length } \\
\qquad(K=\infty)\end{array}$} & \multicolumn{2}{|c|}{ Max. power } & $\frac{N C(P)}{1-\epsilon}$ & $-\sqrt{N \frac{4 C(P) \ln J(P)}{1-\epsilon}} \quad$ (Theorem 4) & $+O(1) \quad([11])$ \\
\hline & \multicolumn{2}{|c|}{ Ave. power } & $\frac{N C(P)}{1-\epsilon}$ & $-\ln N$ & $+O(1) \quad([11])$ \\
\hline
\end{tabular}

impose a maximal power constraint at each possible decoding time as well as average decoding time and average error probability constraints. Definition 1, below, formalizes our code description.

Definition 1: Fix $\epsilon \in(0,1)$, positive scalars $N$ and $P$, and non-negative integers $n_{1}<\ldots<n_{K}$ and $M$. An $\left(N,\left\{n_{i}\right\}_{i=1}^{K}, M, \epsilon, P\right)$ VLSF code comprises

1) a finite alphabet $\mathcal{U}$ and a probability distribution $P_{U}$ on $\mathcal{U}$ defining a common randomness random variable $U$ that is revealed to both the transmitter and the receiver before the start of the transmission, ${ }^{2}$

2) a sequence of encoding functions $\mathrm{f}_{n}: \mathcal{U} \times[M] \rightarrow \mathbb{R}$, $n=1, \ldots, n_{K}$ that assign a codeword

$$
\mathrm{f}(u, m)^{n_{K}} \triangleq\left(\mathrm{f}_{1}(u, m), \ldots, \mathrm{f}_{n_{K}}(u, m)\right)
$$

to each message $m \in[M]$ and common randomness instance $u \in \mathcal{U}$. Each codeword satisfies the nested maximal power constraint $P$ on all sub-codewords, giving

$$
\left\|\mathrm{f}(u, m)^{n_{k}}\right\|^{2} \leq n_{k} P \quad \forall m \in[M], u \in \mathcal{U}, k \in[K],
$$

3) a non-negative integer-valued random stopping time $\tau \in$ $\left\{n_{1}, \ldots, n_{K}\right\}$ for the filtration generated by $\left\{U, Y^{n_{i}}\right\}_{i=1}^{K}$ that satisfies an average decoding time constraint

$$
\mathbb{E}[\tau] \leq N
$$

4) $K$ decoding functions $\mathrm{g}_{n_{k}}: \mathcal{U} \times \mathbb{R}^{n_{k}} \rightarrow[M]$ for $k \in[K]$, satisfying an average error probability constraint

$$
\mathbb{P}\left[\mathrm{g}_{\tau}\left(U, Y^{\tau}\right) \neq W \mid X^{\tau}=f(U, W)^{\tau}\right] \leq \epsilon,
$$

where the message $W$ is uniformly distributed on the set $[M]$.

Random variable $U$ is common randomness shared by the transmitter and receiver. As in [6], [13], [19], the traditional

${ }^{2}$ The realization $u$ of $U$ specifies the codebook. random-coding argument does not prove the existence of a single (deterministic) code that simultaneously satisfies two conditions on the code (e.g., (9) and (10)). Therefore, randomized codes are necessary for our achievability argument; here, $|\mathcal{U}| \leq 2$ suffices [19, Appendix D].

The average power constraint on length- $n_{K}$ codewords in Truong and Tan's VLSF code [12, Def. 1] is given by

$$
\begin{aligned}
\mathbb{E}\left[\left\|\mathrm{f}(U, W)^{n_{K}}\right\|^{2}\right] & =\frac{1}{M} \sum_{m=1}^{M} \sum_{i=1}^{n_{K}} \mathbb{E}\left[\left(\mathrm{f}_{i}(U, m)\right)^{2}\right] \\
& \leq N P .
\end{aligned}
$$

As noted in [12, eq. (100)-(103)], for any code with stopping time $\tau$, the expected value on the left-hand side of (11) can be replaced by the expected value $\mathbb{E}\left[\left\|\mathrm{f}(U, W)^{\tau}\right\|^{2}\right]$ because setting the symbols after time-slot $\tau$ to 0 does not affect the average error probability. Taking the expected value of (8) with respect to stopping time $\tau \in\left\{n_{1}, \ldots, n_{K}\right\}$ and message $W$ shows that any code that satisfies our maximal power constraint (8) also satisfies the average power constraint (12).

We define the maximum achievable message size $M^{*}(N, K, \epsilon, P)$ with $K$ decoding times and nested maximal power constraint $P$ as

$$
\begin{gathered}
M^{*}(N, K, \epsilon, P)=\max \left\{M: \text { an }\left(N,\left\{n_{i}\right\}_{i=1}^{K}, M, \epsilon, P\right)\right. \\
\text { VLSF code exists }\} .
\end{gathered}
$$

An $\left(N,\left\{n_{i}\right\}_{i=1}^{K}, M, \epsilon, P\right)_{\text {ave }}$ VLSF code satisfying an average power constraint and its corresponding maximum achievable message size $M^{*}(N, K, \epsilon, P)$ ave are defined analogously by replacing the maximal power constraint (8) by the average power constraint (12).

\section{Related Work}

The following discussion summarizes prior asymptotic expansions of the maximum achievable message size for the Gaussian channel. 
1) $M^{*}(N, 1, \epsilon, P)$ : For $K=1, P>0$, and $\epsilon \in(0,1)$, Tan and Tomamichel [17, Th. 1] and Polyanskiy et al. [7, Th. 54] show that

$$
\begin{aligned}
& \ln M^{*}(N, 1, \epsilon, P) \\
& =N C(P)-\sqrt{N V(P)} Q^{-1}(\epsilon)+\frac{1}{2} \ln N+O(1) .
\end{aligned}
$$

The converse for (14) is derived [7, Th. 54] and the achievability for (14) in [17, Th. 1], which generates i.i.d. codewords uniformly distributed on the $n$-dimensional sphere with radius $\sqrt{n P}$, and applies maximum likelihood (ML) decoding. These results imply that random codewords uniformly distributed on a sphere and ML decoding are, together, third-order optimal, meaning that the gap between the achievability and converse bounds in (14) is $O(1)$.

2) $M^{*}(N, 1, \epsilon, P)_{\text {ave }}$ For $K=1$ with an average-powerconstraint, Yang et al. show in [18] that

$$
\begin{gathered}
\ln M^{*}(N, 1, \epsilon, P)_{\text {ave }}=N C\left(\frac{P}{1-\epsilon}\right)- \\
-\sqrt{N \ln N V\left(\frac{P}{1-\epsilon}\right)}+O(\sqrt{N}) .
\end{gathered}
$$

Yang et al. use a power control argument to show the achievability of (15). They divide the messages into disjoint sets $\mathcal{A}$ and $[M] \backslash \mathcal{A}$, where $|\mathcal{A}|=$ $M(1-\epsilon)(1-o(1))$. For the messages in $\mathcal{A}$, they use an $\left(N,\{N\},|\mathcal{A}|, \frac{2}{\sqrt{N \ln N}}, \frac{P}{1-\epsilon}(1-o(1))\right)$ VLSF code with a single decoding time $N$. The codewords are generated i.i.d. uniformly on the sphere with radius $\sqrt{N \frac{P}{1-\epsilon}(1-o(1))}$. The messages in $[M] \backslash \mathcal{A}$ are assigned the all-zero codeword. The converse for (15) follows from an application of the meta-converse [7, Th. 26].

3) $M^{*}(N, \infty, \epsilon, P)_{\text {ave }}$ : For VLSF codes with $K=\infty, n_{i}=$ $i-1$ for all $i$, and average power constraint (12), Truong and Tan show in [11, Th. 1] that for any $\epsilon \in(0,1)$ and $P>0$,

$$
\begin{aligned}
& \ln M^{*}(N, \infty, \epsilon, P)_{\text {ave }} \geq \frac{N C(P)}{1-\epsilon}-\ln N+O(1) \\
& \ln M^{*}(N, \infty, \epsilon, P)_{\text {ave }} \leq \frac{N C(P)}{1-\epsilon}+\frac{h_{b}(\epsilon)}{1-\epsilon},
\end{aligned}
$$

where $h_{b}(\epsilon) \triangleq-\epsilon \ln \epsilon-(1-\epsilon) \ln (1-\epsilon)$ is the binary entropy function (in nats). The bounds in (16)-(17) indicate that the $\epsilon$-capacity (the first-order achievable term) is

$$
\lim \inf _{N \rightarrow \infty} \frac{1}{N} \ln M^{*}(N, K, \epsilon, P)=\frac{C(P)}{1-\epsilon} .
$$

The achievable dispersion term is zero, i.e., the secondorder term in the fundamental limit in (16)-(17) is $o(\sqrt{N})$. The results in (16)-(17) are analogous to the fundamental limits for DMCs [6, Th. 2] and follow from arguments similar to those in [6]. Since the information density $\imath(X ; Y)$ for the Gaussian channel is unbounded, bounding the expected value of the decoding time in the proof of [11, Th. 1] requires different techniques from those applicable to DMCs [6].

\section{MAIN RESUlT}

Our main result is an asymptotic achievability bound for the scenario where $K \geq 2$ decoding times are available.

Theorem 1: Fix a finite integer $K \geq 2$ and real numbers $P>0$ and $\epsilon \in(0,1)$. For the Gaussian channel (3) with noise variance 1 and $N$ sufficiently large, the maximum message size (13) achievable by $\left(N,\left\{n_{i}\right\}_{i=1}^{K}, M, \epsilon, P\right)$ VLSF codes satisfies

$$
\begin{aligned}
\ln M^{*}(N, K, \epsilon, P) & \geq \frac{N C(P)}{1-\epsilon}-\sqrt{N \ln _{(K-1)}(N) \frac{V(P)}{1-\epsilon}} \\
& +O\left(\sqrt{\frac{N}{\ln _{(K-1)}(N)}}\right) .
\end{aligned}
$$

Proof: See Section IV-D.

As noted previously, any $\left(N,\left\{n_{i}\right\}_{i=1}^{K}, M, \epsilon, P\right)$ VLSF code is also an $\left(N,\left\{n_{i}\right\}_{i=1}^{\infty}, M, \epsilon, P\right)_{\text {ave }}$ VLSF code. Therefore, (17) provides an upper bound on $\ln M^{*}(N, K, \epsilon, P)$. Theorem 1 and the converse bound in (17) together imply that the $\epsilon$ capacity (18) achievable within the class of VLSF codes in Def. 1 is $\frac{C(P)}{1-\epsilon}$. Neither switching from the average to the maximal power constraint nor limiting the number of decoding times to finite $K$ changes the first-order term. In fact, the same $\epsilon$-capacity is achievable by a variable-length code without feedback that decodes at time 0 with probability $\epsilon(1-o(1))$ (see the achievability proof of [6, Th. 1]). However, comparing Theorem 1 and (16), we see that the second-order term of our new achievability bound is significantly worse than the earlier results. Whether this is the consequence of our tighter power constraint and finite $K$ or a weakness of our code construction is a topic for future research.

In the achievability bound in Theorem 1 , the order of the second-order term, $-\sqrt{N \ln _{(K-1)}(N) \frac{V(P)}{1-\epsilon}}$, depends on the number of decoding times $K$. The larger $K$ grows, the achievable rate converges to the capacity. However, the dependence on $K$ is weak since $\ln _{(K-1)}(N)$ grows very slowly in $N$ even when $K$ is small. For example, for $K=4$ and $N=1000$, $\ln _{(K-1)}(N) \approx 0.659$. Furthermore, for $\epsilon=10^{-3}, P=1$, and $N=1000,83.6 \%$ of the $\epsilon$-capacity is achieved with $K=1$, $85.3 \%$ with $K=2,92.2 \%$ with $K=3$, and $95.4 \%$ with $K=4$. The achievability bounds for $K \in[4]$ and the converse bound (17) are illustrated in Fig. 1.

Theorem 1 builds on the following achievability bound for an $\left(N,\left\{n_{i}\right\}_{i=1}^{K}, M, \frac{1}{\sqrt{N \ln N}}, P\right)$ VLSF code.

Theorem 2: Fix an integer $K \geq 1$ and a real number $P>0$. For the Gaussian channel (3) with noise variance 1 and $N$ sufficiently large, the maximum message size (13) achievable by $\left(N,\left\{n_{i}\right\}_{i=1}^{K}, M, \frac{1}{\sqrt{N \ln N}}, P\right)$ VLSF codes satisfies

$$
\begin{aligned}
& \ln M^{*}\left(N, K, \frac{1}{\sqrt{N \ln N}}, P\right) \geq N C(P) \\
& -\sqrt{N \ln _{(K)}(N) V(P)}+O\left(\sqrt{\frac{N}{\ln _{(K)}(N)}}\right) .
\end{aligned}
$$

Proof: See Section IV-B.

The $K=1$ case in Theorem 2 is recovered by [20, Th. 7], which investigates the moderate deviations regime in channel coding. 


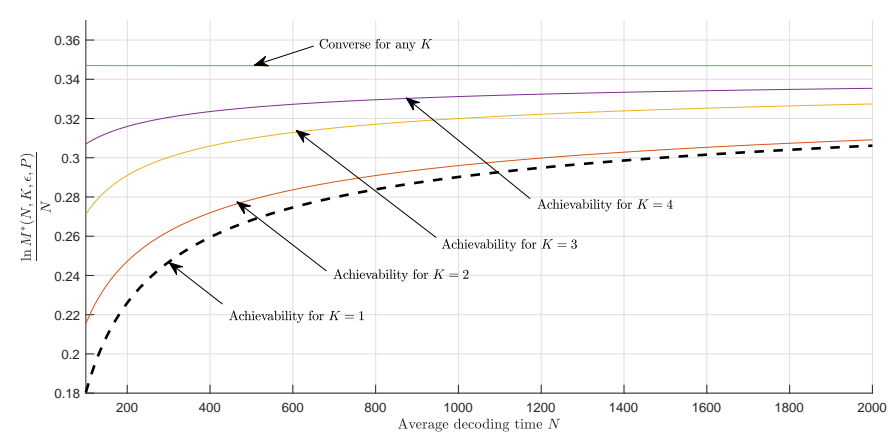

Fig. 1. The achievability (Theorem 1) and converse (17) bounds for the maximum achievable rate $\frac{\ln M^{*}(N, K, \epsilon, P)}{N}$ are shown for $K=1$ (14) and $K \in\{2,3,4\}$ (Theorem 1 ), $P=1$ and $\epsilon=10^{-3}$. The achievability bounds use the asymptotic approximation, i.e., we ignore the $O(\cdot)$ term in (19).

Theorems 1 and 2 follow from an application of the nonasymptotic achievability bound in Theorem 3 , below.

Theorem 3: Fix a constant $\gamma$ and decoding times $n_{1}<\cdots<$ $n_{K}$. For any positive numbers $N, P$, and $\epsilon \in(0,1)$, there exists an $\left(N,\left\{n_{i}\right\}_{i=1}^{K}, M, \epsilon, P\right)$ VLSF code for the Gaussian channel (3) with

$$
\begin{aligned}
& \epsilon \leq \mathbb{P}\left[\imath\left(X^{n_{K}} ; Y^{n_{K}}\right)<\gamma\right]+(M-1) \exp \{-\gamma\} \\
&+\mathbb{P}\left[\bigcup_{i=1}^{K}\left\{\left\|X^{n_{i}}\right\|^{2}>n_{i} P\right\}\right] \\
& N \leq n_{1}+\sum_{i=1}^{K-1}\left(n_{i+1}-n_{i}\right) \mathbb{P}\left[\bigcap_{j=1}^{i}\left\{\imath\left(X^{n_{j}} ; Y^{n_{j}}\right)<\gamma\right\}\right],
\end{aligned}
$$

where $P_{X^{n_{K}}}$ is a product of distributions of $K$ sub-vectors of lengths $n_{j}-n_{j-1}, j \in[K]$, i.e.,

$$
P_{X^{n_{K}}}\left(x^{n_{K}}\right)=\prod_{j=1}^{K} P_{X_{n_{j-1}+1}^{n_{j}}}\left(x_{n_{j-1}+1}^{n_{j}}\right),
$$

where $n_{0}=0$.

Proof: See Section IV-A.

The following result is an achievability bound for an unbounded number of decoding times, i.e., $K=\infty$ under the maximal power constraint (8).

Theorem 4: Fix real numbers $P>0$ and $\epsilon \in(0,1)$. For the Gaussian channel (3) with noise variance 1 , the maximum achievable message size (13) of an $\left(N,\left\{n_{i}\right\}_{i=1}^{\infty}, M, \epsilon, P\right)$ VLSF code satisfies

$$
\begin{aligned}
\ln M^{*}(N, \infty, \epsilon, P) \geq & \frac{N C(P)}{1-\epsilon}-\sqrt{N \frac{4 C(P) \ln J(P)}{1-\epsilon}} \\
& -\ln N+O(1)
\end{aligned}
$$

where

$$
J(P) \triangleq 27 \sqrt{\frac{\pi}{8}} \frac{1+P}{\sqrt{1+2 P}} .
$$

Although $K=\infty$ enables decoding at every time, we prove Theorem 4 using an achievability argument that employs only times $n_{i}=(i-1) \ell_{N}, i=1,2, \ldots$, where $\ell_{N}=O(\sqrt{N})$.

Proof: See Section IV-F.
While the second-order term $-\sqrt{N \ln _{(K-1)}(N) \frac{V(P)}{1-\epsilon}}$ in Theorem 1 behaves similarly to $-O(\sqrt{N})$ for practical values of $N$, it is worse than $-O(\sqrt{N})$ for any finite $K$. Theorem 4 demonstrates that the second-order term $-O(\sqrt{N})$ is achievable when $K=\infty$.

\section{PROOFS}

We prove Theorems 3, 2, 1, and 4 in that order.

\section{A. Proof of Theorem 3}

The proof of Theorem 3 extends the arguments of the random coding bound in [6, Th. 3] to the scenario where we allow only $K$ decoding times, and each codeword satisfies the list of maximal power constraints in (8). Variations of Theorem 3 are proved in [9] and [14]. Our encoding function generates codewords of length $n_{K}$ that comprise $K$ independent subcodewords. The proof of Theorem 3 extends the analysis in $[9$, Sec. III $]$ to account for the maximal power constraint (8) on the sub-codewords.

Random code design: Fix some $P_{X^{n_{K}}}$ such that (23) holds. Define the common randomness random variable $U$ on $\mathbb{R}^{M n_{K}}$ with the distribution

$$
P_{U}=\underbrace{P_{X}^{n_{K}} \times P_{X}^{n_{K}} \times \cdots \times P_{X}^{n_{K}}}_{M \text { times }} .
$$

The realization of $U$ defines the $M$ length- $n_{K}$ codewords $X_{1}^{n_{K}}, X_{2}^{n_{K}}, \ldots, X_{M}^{n_{K}}$.

Decoder design: Define the information density for message $m$ and decoding time $n_{k}$ as

$$
S_{m, n_{k}} \triangleq \imath\left(X_{m}^{n_{k}} ; Y^{n_{k}}\right) \text { for } m \in[M], k \in[K] .
$$

Denote the set of decoding times by

$$
\mathcal{T} \triangleq\left\{n_{1}, n_{2}, \ldots, n_{K}\right\} .
$$

The decoder makes a decision at the first decoding time $n_{k} \in \mathcal{T}$ that the information density exceeds the threshold, i.e., $S_{m, n_{k}} \geq \gamma$ for some message $m \in[M]$. If $S_{m, n_{t}}<\gamma$ for all $m \in[M]$ and $n_{t} \in \mathcal{T}$, then none of the information densities ever reaches the threshold, i.e., the decision is made at time $n_{K}$. The decoding time $\tau^{*}$ is given by

$$
\tau^{*} \triangleq \begin{cases}n_{k} & \text { if } \exists m \in[M] \text { s.t. } S_{m, n_{k}} \geq \gamma, \text { and } \\ & S_{m, n_{t}}<\gamma \forall m \in[M], n_{t} \in \mathcal{T}, t \in[k-1] \\ n_{K} \quad \text { if } S_{m, n_{t}}<\gamma \forall m \in[M], n_{t} \in \mathcal{T} .\end{cases}
$$

If there exists some $m \in[M]$ such that $S_{m, \tau^{*}} \geq \gamma$, then the decoder's output at time $\tau^{*}$ is

$$
\mathrm{g}_{\tau^{*}}\left(U, Y^{n_{\tau^{*}}}\right) \triangleq \max \left\{m \in[M]: S_{m, \tau^{*}} \geq \gamma\right\},
$$

and otherwise, the decoder outputs an arbitrary message.

Expected decoding time analysis: Define the stopping times

$$
\tau_{m} \triangleq \inf \left\{n_{k} \in \mathcal{T}: S_{m, n_{k}} \geq \gamma\right\}, \quad \forall m \in[M] .
$$

When the set over which we take the infimum is empty, we define the infimum to be equal $\infty$. For example, in (31), the case $\tau_{m}=\infty$ signifies that the information density of the 
message $m$ never reaches the threshold, i.e., $S_{m, n_{k}}<\gamma$ for all $n_{k} \in \mathcal{T}$.

The expected decoding time is bounded as

$$
\begin{aligned}
\mathbb{E}\left[\tau^{*}\right] & =\mathbb{E}\left[\min _{m \in[M]} \tau_{m} \wedge n_{K}\right] \\
& \leq \mathbb{E}\left[\tau_{1} \wedge n_{K} \mid W=1\right] \\
& =\sum_{n=0}^{\infty} \mathbb{P}\left[\tau_{1} \wedge n_{K}>n \mid W=1\right] \\
& =n_{1}+\sum_{i=1}^{K-1}\left(n_{i+1}-n_{i}\right) \mathbb{P}\left[\tau_{1}>n_{i} \mid W=1\right],
\end{aligned}
$$

which is equal to the upper bound (22) on the average decoding time.

Error probability analysis: Denote

$$
\begin{aligned}
& \tau \triangleq \inf \left\{n_{k} \in \mathcal{T}: \imath\left(X^{n_{k}} ; Y^{n_{k}}\right) \geq \gamma\right\} \\
& \bar{\tau} \triangleq \inf \left\{n_{k} \in \mathcal{T}: \imath\left(\bar{X}^{n_{k}} ; Y^{n_{k}}\right) \geq \gamma\right\},
\end{aligned}
$$

where $\bar{X}^{n_{K}}$ is any codeword other than the transmitted $X^{n_{K}}$ and therefore is independent of $Y^{n_{K}}$, i.e.,

$$
\begin{aligned}
& P_{X^{n_{K}} \bar{X}^{n_{K}} Y^{n_{K}}}\left(x^{n_{K}}, \bar{x}^{n_{K}}, y^{n_{K}}\right) \\
& \quad=P_{X}^{n_{K}}\left(x^{n_{K}}\right) P_{X}^{n_{K}}\left(\bar{x}^{n_{K}}\right) P_{Y^{n_{K}} \mid X^{n_{K}}}\left(y^{n_{K}} \mid x^{n_{K}}\right) .
\end{aligned}
$$

The error probability is bounded as

$$
\begin{aligned}
\epsilon \leq & \mathbb{P}\left[g_{\tau^{*}}\left(U, Y^{n_{\tau^{*}}}\right) \neq 1 \mid W=1\right] \\
\leq & \mathbb{P}\left[\bigcup_{m=2}^{M}\left\{\tau_{m} \leq \tau_{1}<\infty\right\} \bigcup\left\{\tau_{1}=\infty\right\}\right. \\
& \left.\bigcup\left\{\bigcup_{i=1}^{K}\left\{\left\|X_{1}^{n_{i}}\right\|^{2}>n_{i} P\right\}\right\} \mid W=1\right] \\
\leq & \mathbb{P}\left[\bigcup_{m=2}^{M}\left\{\tau_{m}<\infty\right\} \mid W=1\right]+\mathbb{P}\left[\tau_{1}=\infty \mid W=1\right] \\
& +\mathbb{P}\left[\bigcup_{i=1}^{K}\left\{\left\|X_{1}^{n_{i}}\right\|^{2}>n_{i} P\right\} \mid W=1\right] \\
\leq & (M-1) \mathbb{P}[\bar{\tau}<\infty]+\mathbb{P}[\tau=\infty] \\
& +\mathbb{P}\left[\bigcup_{i=1}^{K}\left\{\left\|X^{n_{i}}\right\|^{2}>n_{i} P\right\}\right]
\end{aligned}
$$

where (39)-(40) follow from the definition of the decoder in (30). In (40), the event that the codeword for message $W=1$ violates the power constraint (8) at any decoding time is treated as an error. Inequality (42) follows by the union bound and that given $W=1, \tau_{1}$ is distributed the same as $\tau$, and $\tau_{m}$, $m \neq 1$ is distributed the same as $\bar{\tau}$. Using the same arguments as in [6, eq. (111)-(118)], we have

$$
\begin{aligned}
\mathbb{P}[\bar{\tau}<\infty] & =\mathbb{E}\left[\exp \left\{-\imath\left(X^{n_{K}} ; Y^{n_{K}}\right)\right\} 1\{\tau<\infty\}\right] \\
& =\mathbb{E}\left[\exp \left\{-\imath\left(X^{\tau} ; Y^{\tau}\right)\right\} 1\{\tau<\infty\}\right] \\
& \leq \exp \{-\gamma\},
\end{aligned}
$$

where (43) follows from changing measure from $P_{\bar{X}^{n} K Y^{n_{K}}}$ to $P_{X^{n_{K}} Y^{n_{K}}}$, (44) follows since $\left\{\exp \left\{-\imath\left(X^{n_{k}} ; Y^{n_{k}}\right)\right\}: n_{k} \in \mathcal{T}\right\} \quad$ is a martingale due to the product distribution in (38), and (45) follows from the definition of $\tau$ in (36). The second probability in (42) is bounded as

$$
\begin{aligned}
\mathbb{P}[\tau=\infty] & =\mathbb{P}\left[\bigcap_{i=1}^{K}\left\{\imath\left(X^{n_{i}} ; Y^{n_{i}}\right)<\gamma\right\}\right] \\
& \leq \mathbb{P}\left[\imath\left(X^{n_{K}} ; Y^{n_{K}}\right)<\gamma\right],
\end{aligned}
$$

where (46) follows from (36). Combining (42), (45), and (47) completes the proof of Theorem 3 .

\section{B. Proof of Theorem 2}

In the proof that follows, we first present three lemmas used in the proof of Theorem 2 (step 1), we then choose the distribution $P_{X}^{n_{K}}$ of the random codewords (step 3) and the parameters $n_{1}, \ldots, n_{K}, \gamma$ in Theorem 3 (step 4). Finally, we analyze the bounds in Theorem 3 under the chosen distribution and parameters (step 5).

1) Supporting lemmas: Lemma 1, below, is the moderate deviations result that bounds the probability that a sum of $n$ i.i.d. random variables is above a function of $n$ that grows at most as quickly as $n^{2 / 3}$.

Lemma 1 (Petrov [21, Ch. 8, Th. 4]): Let $Z_{1}, \ldots, Z_{n}$ be i.i.d. random variables. Let $E\left[Z_{1}\right]=0, \sigma^{2}=\operatorname{Var}\left[Z_{1}\right]$, and $\mu_{3}=\mathbb{E}\left[Z_{1}^{3}\right]$. Suppose that the moment generating function $\mathbb{E}[\exp \{t Z]\}$ is finite in the neighborhood of $t=0$. (This condition is known as Cramer's condition.) Given a nonnegative $z_{n}=O\left(n^{1 / 6}\right)$, as $n \rightarrow \infty$ it holds that

$$
\begin{aligned}
\mathbb{P}\left[\sum_{i=1}^{n} Z_{i} \geq z_{n} \sigma \sqrt{n}\right]= & Q\left(z_{n}\right) \exp \left\{\frac{z_{n}^{3} \mu_{3}}{6 \sqrt{n} \sigma^{3}}\right\} \\
& +O\left(\frac{1}{\sqrt{n}} \exp \left\{-\frac{z_{n}^{2}}{2}\right\}\right) .
\end{aligned}
$$

Lemma 2, below, uniformly bounds the Radon-Nikodym derivative of the channel output distribution in response to the uniform distribution on a sphere compared to the channel output distribution in response to i.i.d. Gaussian distribution.

Lemma 2 (MolavianJazi and Laneman [22, Prop. 2]): Let $X^{n}$ be distributed uniformly over the $n$-dimensional sphere with radius $\sqrt{n P}, \mathbb{S}^{n}(\sqrt{n P})$. Let $\tilde{X}^{n} \sim \mathcal{N}\left(\mathbf{0}, P \mathrm{I}_{n}\right)$. Let $P_{Y^{n}}$ and $P_{\tilde{Y}^{n}}$ denote the channel output distributions in response to $P_{X^{n}}$ and $P_{\tilde{X}^{n}}$, respectively, where $P_{Y^{n} \mid X^{n}}$ is the point-topoint Gaussian channel (3). Then there exists an $n_{0} \in \mathbb{N}$ such that for all $n \geq n_{0}$ and $y^{n} \in \mathbb{R}^{n}$, it holds that

$$
\frac{P_{Y^{n}}\left(y^{n}\right)}{P_{\tilde{Y}^{n}}\left(y^{n}\right)} \leq J(P) \triangleq 27 \sqrt{\frac{\pi}{8}} \frac{1+P}{\sqrt{1+2 P}} .
$$

The following lemma gives the asymptotic expansion of the root of an equation, which is used to find the asymptotic expansion for the gap between two consecutive decoding times $n_{i}$ and $n_{i+1}$.

Lemma 3: Let $f(x)$ be a differentiable increasing function that satisfies $f^{\prime}(x) \rightarrow 0$ as $x \rightarrow \infty$. Suppose that

$$
x+f(x)=y .
$$

Then, as $x \rightarrow \infty$ it holds that

$$
x=y-f(y)(1-o(1)) .
$$


Proof of Lemma 3: Define the function $F(x) \triangleq x+f(x)-y$. Applying Newton's method with the starting point $x_{0}=y$ yields

$$
\begin{aligned}
x_{1} & =x_{0}-\frac{F\left(x_{0}\right)}{F^{\prime}\left(x_{0}\right)} \\
& =y-\frac{f(y)}{1+f^{\prime}(y)} \\
& =y-f(y)\left(1-f^{\prime}(y)+O\left(f^{\prime}(y)^{2}\right) .\right.
\end{aligned}
$$

We have $f^{\prime}(y)=o(1)$ by assumption. Equality (54) follows from the Taylor series expansion of the function $\frac{1}{1+x}$ around $x=0$. Let

$$
x^{\star}=y-f(y)(1-o(1)) .
$$

From Taylor's theorem, it follows that

$$
f\left(x^{\star}\right)=f(y)-f^{\prime}\left(y_{0}\right) f(y)(1-o(1)),
$$

for some $y_{0} \in[y-f(y)(1-o(1)), y]$. Therefore, $f^{\prime}\left(y_{0}\right)=$ $o(1)$, and $f\left(x^{\star}\right)=f(y)(1-o(1))$. Putting (55)-(56) in (50), we see that $x^{\star}$ is a solution to the equality in (50).

2) Random encoder design: To prove Theorem 2, we choose the distribution of the random codewords, $P_{X^{n_{K}}}$, in Theorem 3 as follows. Set $n_{0}=0$. For each codeword, we independently draw sub-codewords $X_{n_{j-1}+1}^{n_{j}}, j \in[K]$ from the uniform distribution on the $\left(n_{j}-n_{j-1}\right)$-dimensional sphere with radius $\sqrt{\left(n_{j}-n_{j-1}\right) P}$, i.e.,

$$
\begin{aligned}
P_{X^{n_{K}}}\left(x^{n_{K}}\right) & =\prod_{j=1}^{K} P_{X_{n_{j-1}+1}^{n_{j}}}\left(x_{n_{j-1}+1}^{n_{j}}\right) \\
P_{X_{n_{j-1}+1}^{n_{j}}}\left(x_{n_{j-1}+1}^{n_{j}}\right) & =\frac{\delta\left(\left\|x_{n_{j-1}+1}^{n_{j}}\right\|^{2}-\left(n_{j}-n_{j-1}\right) P\right)}{S_{\left(n_{j}-n_{j-1}\right)}\left(\sqrt{\left(n_{j}-n_{j-1}\right) P}\right)},
\end{aligned}
$$

where $\delta(\cdot)$ is the Dirac delta function, and

$$
S_{n}(r)=\frac{2 \pi^{n / 2}}{\Gamma(n / 2)} r^{n-1}
$$

is the surface area of an $n$-dimensional sphere $\mathbb{S}^{n}(r)$ with radius $r$. Notice that codewords chosen under the input distribution in (57) never violate the power constraint (8), giving

$$
\mathbb{P}\left[\bigcup_{i=1}^{K}\left\{\left\|X^{n_{i}}\right\|^{2}>n_{i} P\right\}\right]=0 .
$$

Our random coding design distributes codewords uniformly at random on the subset of the surface of $\mathbb{S}^{n_{K}}\left(\sqrt{n_{K} P}\right)$ that satisfies the power constraint with equality at each of the dimensions $n_{1}, \ldots, n_{K}$; our analysis in [23] shows that for any finite $K$, and sufficiently large increments $n_{i}-n_{i-1}$ for all $i \in[K]$, using this restricted subset instead of the entire $n_{K}$-dimensional power sphere results in no change in the asymptotic expansion (14) up to the third-order term.

From Shannon's work in [24], it is well-known that for the Gaussian channel with a maximal power constraint, drawing i.i.d. Gaussian codewords yields a performance inferior to that achieved by the uniform distribution on the power sphere. As a result, almost all tight achievability bounds for the Gaussian channel in the fixed-length regime under a variety of settings (e.g., all four combinations of the maximal/average power constraint and feedback/no feedback [8], [16]-[18] in Table I) employ random codewords drawn uniformly on the power sphere. A notable exception is Truong and Tan's result in (16) $[11$, Th. 1] for VLSF codes with an average power constraint; that result employs i.i.d. Gaussian inputs. The Gaussian distribution works in this scenario because when $K=\infty$, the usually dominant term $\mathbb{P}\left[\imath\left(X^{n_{K}} ; Y^{n_{K}}\right)<\gamma\right]$ in (21) disappears. The second term $(M-1) \exp \{-\gamma\}$ in (21) is not affected by the input distribution. Unfortunately, the approach from [11, Th. 1] does not work here since drawing codewords i.i.d. $\mathcal{N}(0, P)$ satisfies the average power constraint (12) but not the maximal power constraint (8). When $K<\infty$ and the probability $\mathbb{P}\left[\imath\left(X^{n_{K}} ; Y^{n_{K}}\right)<\gamma\right]$ dominates, using i.i.d. $\mathcal{N}(0, P)$ inputs achieves a worse second-order term in the asymptotic expansion (19) of the maximum achievable message size. This implies that when $K<\infty$, using our uniform distribution on a subset of the power sphere is superior to choosing codewords i.i.d. $\mathcal{N}(0, P)$ even under the average power constraint. In particular, i.i.d. $\mathcal{N}(0, P)$ inputs achieve (19), where the dispersion $V(P)$ is replaced by the variance $\tilde{V}(P)=\frac{P}{1+P}$ of $\imath(X ; Y)$ when $X \sim \mathcal{N}(0, P)$; here $\tilde{V}(P)$ is greater than the dispersion $V(P)$ for all $P>0$ (see [25, eq. (2.56)]). Whether our input distribution is optimal in the second-order term remains an open question.

3) Bounding the probability of the information density random variable: For each $k \in[K]$, we here bound the probability

$$
\mathbb{P}\left[\imath\left(X^{n_{k}} ; Y^{n_{k}}\right)<\gamma\right]
$$

that appears in Theorem 3 under the input distribution (57). Note that the random variable $\imath\left(X^{n_{k}} ; Y^{n_{k}}\right)$ is not a sum of $n_{k}$ i.i.d. random variables. We wish to apply the moderate deviations result in Lemma 1. To do this, we first bound the probability in (61) as follows. Let $P_{\tilde{Y}}^{n_{k}}$ be $\mathcal{N}\left(\mathbf{0},(1+P) \mathbf{I}_{n_{k}}\right)$. By Lemma 2, we bound (61) as

$$
\begin{aligned}
& \mathbb{P}\left[\imath\left(X^{n_{k}} ; Y^{n_{k}}\right)<\gamma\right] \\
& =\mathbb{P}\left[\ln \frac{P_{Y^{n_{k}} \mid X^{n_{k}}}\left(Y^{n_{k}} \mid X^{n_{k}}\right)}{P_{\tilde{Y}^{n_{k}}}\left(Y^{n_{k}}\right)}<\gamma+\ln \frac{P_{Y^{n_{k}}}\left(Y^{n_{k}}\right)}{P_{\tilde{Y}^{n_{k}}}\left(Y^{n_{k}}\right)}\right] \\
& \leq \mathbb{P}\left[\ln \frac{P_{Y^{n_{k}} \mid X^{n_{k}}}\left(Y^{n_{k}} \mid X^{n_{k}}\right)}{P_{\tilde{Y}^{n_{k}}}\left(Y^{n_{k}}\right)}<\gamma+k \ln J(P)\right],
\end{aligned}
$$

where $J(P)$ is the constant given in (49), and (63) follows from the fact that $P_{Y^{n_{k}}}$ is product of $k$ output distributions with dimensions $n_{j}-n_{j-1}, j \in[K]$, each induced by a uniform distribution over a sphere with the corresponding radius. As argued in [7], [17], [22], [23], by spherical symmetry, the distribution of the random variable

$$
\ln \frac{P_{Y^{n_{k}} \mid X^{n_{k}}}\left(Y^{n_{k}} \mid X^{n_{k}}\right)}{P_{\tilde{Y}^{n_{k}}}\left(Y^{n_{k}}\right)}
$$


depends on $X^{n_{k}}$ only through its norm $\left\|X^{n_{k}}\right\|$. Since $\left\|X^{n_{k}}\right\|^{2}=n_{k} P$ with probability 1 , any choice of $x^{n_{k}}$ such that $\left\|x^{n_{i}}\right\|^{2}=n_{i} P$ for $i \in[k]$ gives

$$
\begin{aligned}
& \mathbb{P}\left[\ln \frac{P_{Y^{n_{k}} \mid X^{n_{k}}}\left(Y^{n_{k}} \mid X^{n_{k}}\right)}{P_{\tilde{Y}^{n_{k}}}\left(Y^{n_{k}}\right)}<\gamma+k \ln J(P)\right] \\
& =\mathbb{P}\left[\ln \frac{P_{Y^{n_{k}} \mid X^{n_{k}}}\left(Y^{n_{k}} \mid X^{n_{k}}\right)}{P_{\tilde{Y}^{n_{k}}}\left(Y^{n_{k}}\right)}<\gamma+k \ln J(P) \mid X^{n_{k}}=x^{n_{k}}\right]
\end{aligned}
$$

We set $x^{n_{k}}=(\sqrt{P}, \sqrt{P}, \ldots, \sqrt{P})=\sqrt{P} \mathbf{1}$ to obtain an i.i.d. sum in (65). Given $X^{n_{k}}=\sqrt{P} 1$, the distribution of $\imath\left(X^{n_{k}} ; Y^{n_{k}}\right)$ is the same as the distribution of the sum

$$
\sum_{i=1}^{n_{k}} A_{i}
$$

of $n_{k}$ i.i.d. random variables

$$
A_{i}=C(P)+\frac{P}{2(1+P)}\left(1-Z_{i}^{2}+\frac{2}{\sqrt{P}} Z_{i}\right), \quad i \in\left[n_{k}\right] .
$$

Here $Z_{1}, \ldots, Z_{n_{k}}$ are drawn independently from $\mathcal{N}(0,1)$ (see e.g., [7, eq. (205)]). The mean and variance of $A_{1}$ are

$$
\begin{aligned}
\mathbb{E}\left[A_{1}\right] & =C(P) \\
\operatorname{Var}\left[A_{1}\right] & =V(P) .
\end{aligned}
$$

From (63)-(66), we get

$$
\mathbb{P}\left[\imath\left(X^{n_{k}} ; Y^{n_{k}}\right)<\gamma\right] \leq \mathbb{P}\left[\sum_{i=1}^{n_{k}} A_{i}<\gamma+k \ln J(P)\right] .
$$

To verify that Lemma 1 is applicable to the right-hand side of (70), it only remains to show that $\mathbb{E}\left[\left(A_{1}-C(P)\right)^{3}\right]$ is finite, and $A_{1}-C(P)$ satisfies Cramer's condition, that is, there exists some $t_{0}>0$ such that $\mathbb{E}\left[\exp \left\{t\left(A_{1}-C(P)\right)\right\}\right]<\infty$ for all $|t|<t_{0}$. From (67), $\left(A_{1}-C(P)\right)^{3}$ is distributed the same as a 6-degree polynomial of the Gaussian random variable $Z \sim \mathcal{N}(0,1)$. This polynomial has a finite mean since all moments of $Z$ are finite. Let $c \triangleq \frac{P}{2(1+P)}, d \triangleq \frac{2}{\sqrt{P}}$, and $t^{\prime} \triangleq t c$. To show that Cramer's condition holds, we directly compute

$$
\begin{aligned}
& \mathbb{E}\left[\exp \left\{t\left(A_{1}-C(P)\right)\right\}\right] \\
& =\mathbb{E}\left[\exp \left\{t^{\prime}\left(1-Z^{2}+d Z\right)\right\}\right] \\
& =\int_{-\infty}^{\infty} \frac{1}{\sqrt{2 \pi}} \exp \left\{-\frac{x^{2}}{2}+t^{\prime}\left(1-x^{2}+d x\right)\right\} d x \\
& =\frac{1}{\sqrt{1+2 t^{\prime}}} \exp \left\{t^{\prime}+\frac{t^{\prime} d}{2\left(1+2 t^{\prime}\right)}\right\} .
\end{aligned}
$$

Thus $\mathbb{E}\left[\exp \left\{t\left(A_{1}-C(P)\right)\right\}\right]<\infty$ for $t^{\prime}>-\frac{1}{2}$, and $t_{0}=$ $\frac{1}{2 c}>0$ together imply that $A_{1}-C(P)$ satisfies Cramer's condition.

4) Choosing the decoding times $n_{1}, \ldots, n_{K}$ : We choose $\gamma, n_{1}, \ldots, n_{K}$ so that the equalities

$$
\gamma+K \ln J(P)=n_{k} C(P)-\sqrt{n_{k} \ln _{(K-k+1)}\left(n_{k}\right) V(P)}
$$

hold for all $k \in[K]$. This choice minimizes the upper bound (22) on the average decoding time (see Section IV-C). Applying Lemma 3 to (74) with

$$
\begin{aligned}
x & =n_{i+1} \\
y & =n_{i}-\frac{1}{C(P)} \sqrt{n_{i} \ln _{(K-i+1)}\left(n_{i}\right) V(P)} \\
f(x) & =-\frac{1}{C(P)} \sqrt{n_{i+1} \ln _{(K-i)}\left(n_{i+1}\right) V(P)}
\end{aligned}
$$

gives the following gaps between consecutive decoding times

$$
\begin{aligned}
n_{i+1}-n_{i} & =\frac{1}{C(P)}\left(\sqrt{n_{i} \ln _{(K-i)}\left(n_{i}\right) V(P)}\right. \\
& \left.-\sqrt{n_{i} \ln _{(K-i+1)}\left(n_{i}\right) V(P)}\right)(1+o(1))
\end{aligned}
$$

for $i \in\{1, \ldots, K-1\}$.

5) Analyzing the bounds in Theorem 3: For each $k \in[K]$, applying Lemma 1 to (70) with $\gamma, n_{1}, \ldots, n_{K}$ satisfying (74) gives

$$
\begin{aligned}
\mathbb{P}[ & \left.\imath\left(X^{n_{k}} ; Y^{n_{k}}\right)<\gamma\right] \\
\leq & Q\left(\sqrt{\ln _{(K-k+1)}\left(n_{k}\right)}\right) \\
& \cdot \exp \left\{\frac{-\left(\ln _{(K-k+1)}\left(n_{k}\right)\right)^{3 / 2} \mu_{3}(P)}{6 \sqrt{n} V(P)^{3 / 2}}\right\} \\
& +O\left(\frac{1}{\sqrt{n}} \exp \left\{-\frac{\ln _{(K-k+1)}\left(n_{k}\right)}{2}\right\}\right) \\
\leq & \left.\frac{1}{\sqrt{2 \pi}} \frac{1}{\sqrt{\ln _{(K-k)}\left(n_{k}\right)}} \frac{1}{\sqrt{\ln _{(K-k+1)}\left(n_{k}\right)}}\right) \\
& \cdot\left(1+O\left(\frac{\left(\ln _{(K-k+1)}\left(n_{k}\right)\right)^{(3 / 2)}}{\sqrt{n}}\right)\right.
\end{aligned}
$$

for $k<K$, where

$$
\mu_{3}(P) \triangleq \mathbb{E}\left[\left(A_{1}-C(P)\right)^{3}\right]<\infty
$$

and (80) follows from the Taylor series expansion $\exp (x)=$ $1+x+O\left(x^{2}\right)$ as $x \rightarrow 0$, and the well-known bound [21, e.g., Ch. 8, eq. (2.46)]

$$
Q(x) \leq \frac{1}{\sqrt{2 \pi}} \frac{1}{x} \exp \left\{-\frac{x^{2}}{2}\right\} \quad \text { for } x>0 .
$$

For $k=K$, Lemma 1 gives

$$
\begin{aligned}
& \mathbb{P}\left[\imath\left(X^{n_{K}} ; Y^{n_{K}}\right)<\gamma\right] \\
& \leq \frac{1}{\sqrt{2 \pi}} \frac{1}{\sqrt{n_{K}}} \frac{1}{\sqrt{\ln n_{K}}}\left(1+O\left(\frac{\left(\ln n_{K}\right)^{(3 / 2)}}{\sqrt{n}}\right)\right) .
\end{aligned}
$$

We bound the probability term in (22) from above by $\mathbb{P}\left[\imath\left(X^{n_{i}} ; Y^{n_{i}}\right)<\gamma\right]$. Then, by Theorem 3 , there exists a VLSF code with $K$ decoding times $n_{1}<n_{2}<\cdots<n_{K}$ such that the expected decoding time is bounded as

$$
N \leq n_{1}+\sum_{i=1}^{K-1}\left(n_{i+1}-n_{i}\right) \mathbb{P}\left[\imath\left(X^{n_{i}} ; Y^{n_{i}}\right)<\gamma\right]
$$

By (78), we have

$$
n_{k}=n_{1}(1+o(1))
$$


for $k \in[K]$. Plugging (78), (80), and (85) into (84), we get

$$
N \leq n_{1}+\frac{\sqrt{V(P)}}{\sqrt{2 \pi} C(P)} \frac{\sqrt{n_{1}}}{\sqrt{\ln _{(K)}\left(n_{1}\right)}}(1+o(1)) .
$$

Applying Lemma 3 to (86), we get

$$
n_{1} \geq N-\frac{\sqrt{V(P)}}{2 C(P)} \frac{\sqrt{N}}{\sqrt{\ln (K)}(N)}
$$

for $n_{1}$ large enough. Comparing (87) and (78), we observe that for $n_{1}$ large enough,

$$
n_{1}<N<n_{2}<\cdots<n_{K} .
$$

Finally, we set message size $M$ such that

$$
\ln M=\gamma-\ln N \text {. }
$$

Plugging (83) and (89) into (21), we bound the error probability as

$$
\begin{aligned}
\epsilon & \leq \mathbb{P}\left[\imath\left(X^{n_{K}} ; Y^{n_{K}}\right)<\gamma\right]+(M-1) \exp \{-\gamma\} \\
& \leq \frac{1}{2} \frac{1}{\sqrt{n_{K}}} \frac{1}{\sqrt{\ln n_{K}}}+\frac{1}{N} \\
& \leq \frac{1}{2} \frac{1}{\sqrt{N}} \frac{1}{\sqrt{\ln N}}+\frac{1}{N},
\end{aligned}
$$

where (91) holds for $n_{K}$ large enough and (92) follows from (88). Inequality (92) implies that $\epsilon \leq \frac{1}{\sqrt{N \ln N}}$ for $N$ large enough. Plugging (87) and (89) into (74) with $k=1$, we conclude that there exists an $\left(N,\left\{n_{i}\right\}_{i=1}^{K}, M, \frac{1}{\sqrt{N \ln N}}, P\right)$ VLSF code with

$$
\begin{aligned}
\ln M \geq & N C(P)-\sqrt{N \ln _{(K)}(N) V(P)} \\
& -\frac{1}{2} \sqrt{\frac{N V(P)}{\ln _{(K)}(N)}-\ln N-K \ln J(P)}
\end{aligned}
$$

for $N$ large enough, which completes the proof.

C. Second-order Optimality of $\left(n_{1}, \ldots, n_{K}\right)$ in Theorem 2 within the Chosen Code Construction

We here investigate the optimality of our parameter choices in (74). For a fixed $M$ and the given code construction, we would like to minimize the average decoding time subject to the desired error probability. We consider the optimization problem in Theorem 3, that is,

$$
\begin{aligned}
\min & n_{1}+\sum_{i=1}^{K-1}\left(n_{i+1}-n_{i}\right) \mathbb{P}\left[\imath\left(X^{n_{i}} ; Y^{n_{i}}\right)<\gamma\right] \\
\text { s.t. } & \frac{1}{\sqrt{n_{K} \ln n_{K}}}= \\
& +\left(M\left[\left(X^{n_{K}} ; Y^{n_{K}}\right)<\gamma\right]\right. \\
& +(M-1) \exp \{-\gamma\} .
\end{aligned}
$$

We set $n_{K}$ and $\gamma$ to satisfy the equations

$$
\begin{aligned}
\frac{1}{\sqrt{n_{K} \ln n_{K}}} & =\mathbb{P}\left[\imath\left(X^{n_{K}} ; Y^{n_{K}}\right)<\gamma\right](1+o(1)) \\
\ln M & =\gamma-\ln n_{K} .
\end{aligned}
$$

The optimality of the choices in (95)-(96) are discussed later in Section IV-E.
Given the choices in (95)-(96), the problem reduces to minimizing

$$
\begin{aligned}
& N\left(n_{1}, \ldots, n_{K-1}\right) \\
& \quad=n_{1}+\sum_{i=1}^{K-1}\left(n_{i+1}-n_{i}\right) \mathbb{P}\left[\imath\left(X^{n_{i}} ; Y^{n_{i}}\right)<\gamma\right]
\end{aligned}
$$

for fixed $n_{K}$ and $\gamma$ under the constraints (95)-(96).

Next, we define the functions

$$
\begin{aligned}
& g(n) \triangleq \frac{n C(P)-\gamma}{\sqrt{n V(P)}} \\
& F(n) \triangleq Q(-g(n))=1-Q(g(n)) \\
& f(n) \triangleq F^{\prime}(n)=\frac{1}{\sqrt{2 \pi}} \exp \left\{-\frac{g(n)^{2}}{2}\right\} g^{\prime}(n) .
\end{aligned}
$$

Assume that $\gamma=\gamma_{n}$ is such that $g(n)=O\left(n^{1 / 6}\right)$, and $\lim _{n \rightarrow \infty} g(n)=\infty$. Then by Lemma 1 , the step-wise constant function of $n, \mathbb{P}\left[\imath\left(X^{n} ; Y^{n}\right) \geq \gamma\right]$, is approximated by differentiable function $F(n)$ as

$$
\mathbb{P}\left[\imath\left(X^{n} ; Y^{n}\right) \geq \gamma\right]=F(n)(1+o(1)) .
$$

Taylor series expansions give

$$
\begin{aligned}
1-F(n) & =\frac{1}{g(n)} \frac{1}{\sqrt{2 \pi}} \exp \left\{-\frac{g(n)^{2}}{2}\right\}(1+o(1)) \\
f(n) & =(1-F(n)) g(n) g^{\prime}(n)(1+o(1)) \\
g^{\prime}(n) & =\frac{C(P)}{\sqrt{n V(P)}}(1+o(1)) .
\end{aligned}
$$

Let $\mathbf{n}^{*}=\left(n_{1}^{*}, \ldots, n_{K-1}^{*}\right)$ denote the solution to the optimization problem in (94). Then $\mathbf{n}^{*}$ must satisfy the KarushKuhn-Tucker conditions $\nabla N\left(\mathbf{n}^{*}\right)=\mathbf{0}$, giving

$$
\begin{aligned}
& \left.\frac{\partial N}{\partial n_{1}}\right|_{\mathbf{n}=\mathbf{n}^{*}}=F\left(n_{1}^{*}\right)-\left(n_{2}^{*}-n_{1}^{*}\right) f\left(n_{1}^{*}\right)=0 \\
& \left.\frac{\partial N}{\partial n_{k}}\right|_{\mathbf{n}=\mathbf{n}^{*}}=F\left(n_{k}^{*}\right)-F\left(n_{k-1}^{*}\right)-\left(n_{k+1}^{*}-n_{k}^{*}\right) f\left(n_{k}^{*}\right)=0,
\end{aligned}
$$

for $k=2, \ldots, K-1$.

The method of approximating the probability $\mathbb{P}\left[\imath\left(X^{n} ; Y^{n}\right) \geq \gamma\right]$ by a differentiable function $F(n)$ is introduced in [15, Sec. III] for the optimization problem in (94). In [15], Heidarzadeh et al. consider $F(n)$ as a proxy for the cumulative distribution function of the decoding time random variable $\tau$. They derive the Karush-Kuhn-Tucker conditions in (105)-(106), and use them to bound the performance of the VLSF code performance with $K$ decoding times. The channel considered there is the binary erasure channel, and the analysis employs a random linear coding scheme. The analysis in [15] derives the approximation $F(n)$ and uses it to numerically solve the equations (105)-(106) for a fixed $K, M$, and $\epsilon$. Unlike [15], we find the analytic solution to (105)-(106) for the Gaussian channel within our code construction as decoding times $n_{1}, \ldots, n_{K}$ approach infinity, and derive the achievable rate in Theorem 1 as a function of $K$. 
Let $\tilde{\mathbf{n}}=\left(\tilde{n}_{1}, \ldots, \tilde{n}_{K-1}\right)$ be the decoding times chosen in (74). We evaluate

$$
\begin{aligned}
g\left(\tilde{n}_{i}\right) & =\sqrt{\ln _{(K-i+1)}\left(\tilde{n}_{i}\right)}(1+o(1)) \\
1-F\left(g\left(\tilde{n}_{i}\right)\right) & =\frac{1}{\sqrt{2 \pi}} \frac{1}{g\left(\tilde{n}_{i+1}\right) g\left(\tilde{n}_{i}\right)}(1+o(1)) \\
f\left(g\left(\tilde{n}_{i}\right)\right) & =\frac{1}{\sqrt{2 \pi}} \frac{g^{\prime}\left(\tilde{n}_{i}\right)}{g\left(\tilde{n}_{i+1}\right)} \\
\tilde{n}_{i+1}-\tilde{n}_{i} & =\frac{g\left(\tilde{n}_{i+1}\right)}{g^{\prime}\left(\tilde{n}_{i}\right)}(1+o(1))
\end{aligned}
$$

for $i=1, \ldots, K-1$, and

$$
\begin{aligned}
\nabla N(\tilde{\mathbf{n}})= & \left(1-\frac{1}{\sqrt{2 \pi}},-\frac{1}{\sqrt{2 \pi}},-\frac{1}{\sqrt{2 \pi}}, \ldots,-\frac{1}{\sqrt{2 \pi}}\right) \\
& (1+o(1)) .
\end{aligned}
$$
that

Our goal is to find a vector $\Delta \mathbf{n}=\left(\Delta n_{1}, \ldots, \Delta n_{K-1}\right)$ such

$$
\nabla N(\tilde{\mathbf{n}}+\Delta \mathbf{n})=\mathbf{0},
$$

Assume that $\Delta n=O(\sqrt{n})$. By plugging $n+\Delta n$ into (102)(104) and using the Taylor series expansion of $g(n+\Delta n)$, we get

$$
\begin{aligned}
1-F(n+\Delta n)= & (1-F(n)) \\
& \cdot \exp \left\{-\Delta n g(n) g^{\prime}(n)\right\}(1+o(1)) \\
f(n+\Delta n)= & f(n) \exp \left\{-\Delta n g(n) g^{\prime}(n)\right\}(1+o(1)) .
\end{aligned}
$$

Using (113)-(114), and putting $\tilde{\mathbf{n}}+\Delta \mathbf{n}$ in (105)-(106), we solve (112) as

$$
\begin{aligned}
\Delta n_{1} & =-\frac{\ln \sqrt{2 \pi}}{g\left(\tilde{n}_{1}\right) g^{\prime}\left(\tilde{n}_{1}\right)}(1+o(1)) \\
& =-\frac{\sqrt{V(P)} \ln \sqrt{2 \pi}}{C(P)} \frac{\sqrt{\tilde{n}_{1}}}{\sqrt{\ln _{(K)}\left(\tilde{n}_{1}\right)}}(1+o(1)) \\
\Delta n_{i} & =\frac{1}{2} \frac{g\left(\tilde{n}_{i-1}\right)^{2}}{g\left(\tilde{n}_{i}\right) g^{\prime}\left(\tilde{n}_{i}\right)}=o\left(\Delta n_{1}\right)(1+o(1))
\end{aligned}
$$

for $i=2, \ldots, K-1$. Hence, $\tilde{\mathbf{n}}+\Delta \mathbf{n}$ satisfies the optimality criterion, and $\mathbf{n}^{*}=\tilde{\mathbf{n}}+\Delta \mathbf{n}$.

Now it only remains to evaluate the gap $N\left(\mathbf{n}^{*}\right)-N(\tilde{\mathbf{n}})$. We have

$$
\begin{aligned}
N\left(\mathbf{n}^{*}\right)-N(\tilde{\mathbf{n}}) & \left(\Delta n_{1}+\sum_{i=1}^{K-1}\left(\tilde{n}_{i+1}-\tilde{n}\right) Q\left(g\left(\tilde{n}_{i}\right)\right)\right. \\
& \left.\cdot\left(\exp \left\{-\Delta n_{i} g\left(\tilde{n}_{i}\right) g^{\prime}\left(\tilde{n}_{i}\right)\right\}-1\right)\right)(1+o(1)) \\
= & \left(\Delta n_{1}+\left(1-\frac{1}{\sqrt{2 \pi}}\right) \frac{1}{g\left(\tilde{n}_{1}\right) g^{\prime}\left(\tilde{n}_{i}\right)}-\sum_{i=2}^{K-1} \Delta n_{i}\right) \\
& \cdot(1+o(1)) \\
= & -L \frac{\sqrt{\tilde{n}_{1}}}{\sqrt{\ln _{(K)}\left(\tilde{n}_{1}\right)}}(1+o(1)),
\end{aligned}
$$

where $L=\left(\ln \sqrt{2 \pi}+\frac{1}{\sqrt{2 \pi}}-1\right) \frac{\sqrt{V(P)}}{C(P)}$ is a positive constant. From the relationship between $n_{k}$ and $n_{1}$ in (85) and the equality (120), we get

$$
N(\tilde{\mathbf{n}})=N\left(\mathbf{n}^{*}\right)+L \frac{\sqrt{N\left(\mathbf{n}^{*}\right)}}{\sqrt{\ln _{(K)}\left(N\left(\mathbf{n}^{*}\right)\right)}}(1+o(1)) .
$$

Plugging (121) into our VLSF achievability bound (93), we get

$$
\begin{aligned}
\ln M \geq & N\left(\mathbf{n}^{*}\right) C(P)-\sqrt{N\left(\mathbf{n}^{*}\right) \ln _{(K)}\left(N\left(\mathbf{n}^{*}\right)\right) V(P)} \\
& -O\left(\sqrt{\frac{N\left(\mathbf{n}^{*}\right)}{\ln _{(K)}\left(N\left(\mathbf{n}^{*}\right)\right)}}\right) .
\end{aligned}
$$

Comparing (122) and (93), we see that the decoding times chosen in (74) have the optimal second-order term in the asymptotic expansion of the maximum achievable message size within our code construction. Moreover, the order of the third-order term in (122) is the same as the order of the thirdorder term in (93). Whether the proposed code structure is optimal remains an open question.

\section{Proof of Theorem 1}

Our achievability proof is inspired by Polyanskiy et al.'s coding scheme in [6] for DMCs. There, the number of decoding times is unlimited. In [6], to prove an achievability result with an average error probability $\epsilon$ and an average decoding time $N$, the decoder decodes to an arbitrary message at time $n_{1}=0$ with probability $p=\frac{\epsilon-\epsilon_{N}^{\prime}}{1-\epsilon_{N}^{\prime}}$. With probability $1-p$, the code uses another VLSF code with average decoding time $N^{\prime}$ and average error probability $\epsilon_{N}^{\prime}=\frac{1}{N^{\prime}}$. With the same choice of $\epsilon_{N}^{\prime}$, Truong and Tan [11], [12] adapt the coding scheme in [6] to the Gaussian point-to-point and multiple access channels with an unlimited number of decoding times and an average power constraint. Like [6], [11], [12], our coding strategy fixes the smallest decoding time $n_{1}$ to 0 . To achieve the best secondorder term within our code construction (see Section IV-E, below), we set

$$
\epsilon_{N}^{\prime} \triangleq \frac{1}{\sqrt{N^{\prime} \ln N^{\prime}}}
$$

Before the transmission starts, the decoder generates a random variable $D \sim \operatorname{Bernoulli}(p)$ with

$$
p \triangleq \frac{\epsilon-\epsilon_{N}^{\prime}}{1-\epsilon_{N}^{\prime}} .
$$

If $D=1$, then the decoder decodes to an arbitrary message at time $n_{1}=0$; otherwise the encoder and decoder use an $\left(N^{\prime},\left\{n_{k}\right\}_{k=2}^{K}, M, \epsilon_{N}^{\prime}, P\right)$ VLSF code. The average error probability is bounded by

$$
1 \cdot p+\epsilon_{N}^{\prime} \cdot(1-p)=\epsilon,
$$

and the average decoding time is

$$
N=p \cdot 0+(1-p) \cdot N^{\prime}=(1-p) N^{\prime} .
$$

From (124), and (126), we get the asymptotic expansion

$$
N^{\prime}=\frac{N}{1-\epsilon}\left(1+O\left(\frac{1}{\sqrt{N \ln N}}\right)\right) .
$$


By Theorem 2, there exists an $\left(N^{\prime},\left\{n_{k}\right\}_{k=2}^{K}, M, \epsilon_{N}^{\prime}, P\right)$ VLSF code with

$$
\begin{aligned}
\ln M= & N^{\prime} C(P)-\sqrt{N^{\prime} \ln _{(K-1)}\left(N^{\prime}\right) V(P)} \\
& +O\left(\sqrt{\frac{N^{\prime}}{\ln _{(K-1)}\left(N^{\prime}\right)}}\right) .
\end{aligned}
$$

Plugging (127) into (128) and using the Taylor series expansion of the function $\sqrt{N^{\prime} \ln _{(K-1)}\left(N^{\prime}\right)}$ completes the proof.

E. Second-order Optimality of the Parameter $\epsilon_{N}^{\prime}$ in Section IV-D within the Chosen Code Construction

In this section, we show that the error value $\epsilon_{N}^{\prime}$ chosen in (123) is second-order optimal in the sense that under the VLSF code design in Section IV-D, the error value $\epsilon_{N}^{\prime *}$ that minimizes the average decoding time in (126) has the same asymptotic expansion (19) of the maximum achievable message size as $\epsilon_{N}^{\prime}$ up to the second-order term.

From the code construction in Section IV-D, Theorem 3, (124), and (126), the average decoding time is

$$
N\left(n_{2}, \ldots, n_{K}, \gamma\right)=N^{\prime}(1-\epsilon) \frac{1}{1-\epsilon_{N}^{\prime}},
$$

where

$$
\begin{aligned}
N^{\prime} & =n_{2}+\sum_{i=2}^{K-1}\left(n_{i+1}-n_{i}\right) \mathbb{P}\left[\imath\left(X^{n_{i}} ; Y^{n_{i}}\right)<\gamma\right] \\
\epsilon_{N}^{\prime} & =\mathbb{P}\left[\imath\left(X^{n_{K}} ; Y^{n_{K}}\right)<\gamma\right]+M \exp \{-\gamma\} .
\end{aligned}
$$

Let $\left(\mathbf{n}^{*}, \gamma^{*}\right)=\left(n_{2}^{*}, \ldots, n_{K}^{*}, \gamma^{*}\right)$ be the solution of $\nabla N\left(\mathbf{n}^{*}, \gamma^{*}\right)=\mathbf{0}$. As in Section IV-C, we use the approximation $\mathbb{P}\left[\imath\left(X^{n_{i}} ; Y^{n_{i}}\right)<\gamma\right]=Q\left(g\left(n_{i}\right)\right)(1+o(1))$, where $g(n)$ is given in (98). The prior discussion (105)-(106) ${ }^{3}$ finds the values of $n_{2}^{*}, n_{3}^{*}, \ldots, n_{K-1}^{*}$ that minimize $N^{\prime}$. Minimizing $N^{\prime}$ also minimizes $N$ in (129) since $\epsilon_{N}^{\prime}$ depends only on $n_{K}$ and $\gamma$, and $\epsilon$ is a constant. Therefore, to minimize $N$, it only remains to find $\left(n_{K}^{*}, \gamma^{*}\right)$ such that

$$
\begin{aligned}
& \left.\frac{\partial N}{\partial n_{K}}\right|_{(\mathbf{n}, \gamma)=\left(\mathbf{n}^{*}, \gamma^{*}\right)}=0 \\
& \left.\frac{\partial N}{\partial \gamma}\right|_{(\mathbf{n}, \gamma)=\left(\mathbf{n}^{*}, \gamma^{*}\right)}=0 .
\end{aligned}
$$

Consider the case $K>2$. Solving (132) and (133) using (105)-(110) gives

$$
\begin{aligned}
g\left(n_{K}^{*}\right)= & \sqrt{\ln n_{K}^{*}+\ln _{(2)}\left(n_{K}^{*}\right)+\ln _{(3)}\left(n_{K}^{*}\right)+O(1)} \\
0= & c_{0}+N^{\prime}\left(\frac{1}{\sqrt{2 \pi n_{K}^{*}}} \exp \left\{-\frac{g\left(n_{K}^{*}\right)^{2}}{2}\right\}(1+o(1))\right. \\
& \left.-M \exp \left\{-\gamma^{*}\right\}\right),
\end{aligned}
$$

where $c_{0}$ is a positive constant. Solving (134)-(135) simultaneously, we obtain

$$
\ln M=\gamma^{*}-\ln n_{K}^{*}+O(1) .
$$

\footnotetext{
${ }^{3}$ Due to (130), we invoke the proof in Section IV-C with $K-1$ decoding times instead of $K$.
}

Plugging (134) and (136) into (131), we get

$$
\epsilon_{N}^{\prime *}=\frac{c_{1}}{\sqrt{n_{K}^{*} \ln _{(2)}\left(n_{K}^{*}\right)} \ln n_{K}^{*}}(1+o(1)),
$$

where $c_{1}$ is a constant. Let $(\tilde{\mathbf{n}}, \tilde{\gamma})=\left(\tilde{n}_{2}, \ldots, \tilde{n}_{K}, \tilde{\gamma}\right)$ be the parameters chosen in (74) and (89). Note that $\epsilon_{N}^{\prime *}$ is orderwise different than $\epsilon_{N}^{\prime}$ in (123). Following steps similar to (118)-(120), we compute

$$
N\left(\mathbf{n}^{*}, \gamma^{*}\right)-N(\tilde{\mathbf{n}}, \tilde{\gamma})=-O\left(\frac{\sqrt{n_{K}^{*}}}{\sqrt{\ln n_{K}^{*}}}\right) .
$$

Plugging (138) into (19) gives

$$
\begin{aligned}
\ln M & =\frac{N\left(\mathbf{n}^{*}, \gamma^{*}\right) C(P)}{1-\epsilon} \\
& -\sqrt{N\left(\mathbf{n}^{*}, \gamma^{*}\right) \ln _{(K-1)}\left(N\left(\mathbf{n}^{*}, \gamma^{*}\right)\right) \frac{V(P)}{1-\epsilon}} \\
& +O\left(\sqrt{\frac{N\left(\mathbf{n}^{*}, \gamma^{*}\right)}{\ln _{(K-1)}(N)}}\right)
\end{aligned}
$$

Comparing (19) and (139), we see that although (123) and (137) are different, the parameters $(\tilde{\mathbf{n}}, \tilde{\gamma})$ chosen in (74) and (89) have the same second-order term in the asymptotic expansion of the maximum achievable message size as the parameters $\left(\mathbf{n}^{*}, \gamma^{*}\right)$ that minimize the average decoding time within our code structure.

For $K=2$, the summation term in (131) disappears; in this case, the solution to (132) gives

$$
\epsilon_{N}^{\prime *}=\frac{c_{2}}{\sqrt{n_{K}^{*} \ln n_{K}^{*}}}(1+o(1))
$$

for some constant $c_{2}$. Following the steps in (138)-(139), we conclude that the parameter choices in (74) and (89) are second-order optimal within our code construction for $K=2$ as well.

\section{F. Proof of Theorem 4}

As in the proof of Theorem 1, we first show an achievability result for a vanishing error probability. As in [6], [12], we set the vanishing error probability to be $\frac{1}{N}$.

1) Achievability of VLSF codes with a vanishing error probability:

Lemma 4: Fix $P>0$. For the Gaussian channel (3) with noise variance 1 , it holds that

$$
\begin{aligned}
\ln M^{*}\left(N, \infty, \frac{1}{N}, P\right) \geq & N C(P)-\sqrt{N 4 C(P) \ln J(P)} \\
& -\ln N+O(1) .
\end{aligned}
$$

Proof of Lemma 4: To prove Lemma 4, we apply Theorem 3 with $K=\infty$ and

$$
\mathcal{T}=\left\{n_{k}: k \ell_{N}, k \in \mathcal{Z}^{+}\right\} .
$$

Here $\ell_{N}$ is the gap between consecutive decoding times. That gap is here constant within the given code but grows with the average decoding time $N$ for which the code is designed. How $\ell_{N}$ grows with $N$ is determined later (see (157), below). 
Choosing the input distribution: Let the distribution, $P_{X^{n}{ }_{K}}$, of the random codewords, be chosen as in (57). In this case, $K=\infty$.

Expected decoding time analysis: Following the steps in (32)-(33), we bound the average decoding time as

$$
\mathbb{E}\left[\tau^{*}\right] \leq \mathbb{E}[\tau],
$$

where $\tau$ is given in (36). We define the random variable

$$
\xi \triangleq \inf \left\{k \geq 1: \frac{1}{\ell_{N}} \imath\left(X^{k \ell_{N}} ; Y^{k \ell_{N}}\right)>\frac{\gamma}{\ell_{N}}\right\}=\frac{\tau}{\ell_{N}} .
$$

To bound $\mathbb{E}[\xi]$, we use the following result from the renewal theory literature.

Lemma 5 (Lorden [26, Th. 1]): Let $X_{1}, X_{2}, \ldots$ be i.i.d. random variables with $\mathbb{E}\left[X_{1}\right]=\mu>0$ and $\mathbb{E}\left[(\max \{0, X\})^{2}\right]=$ $m<\infty$. Let $S_{n}=\sum_{i=1}^{n} X_{i}$ and $\tau(\gamma)=\inf \left\{n \in \mathbb{N}: S_{n}>\right.$ $\gamma$ \}. Then,

$$
\sup _{\gamma>0} \mathbb{E}[\tau(\gamma)] \leq \frac{\gamma}{\mu}+\frac{m}{\mu^{2}}
$$

Note that

$$
\frac{1}{\ell_{N}} \imath\left(X^{k \ell_{N}} ; Y^{k \ell_{N}}\right)=\sum_{i=1}^{k} B_{i}
$$

is a sum of $k$ i.i.d. random variables $B_{1}, \ldots, B_{k}$. The expected value of $B_{1}$ is bounded as

$$
\begin{aligned}
\mathbb{E}\left[B_{1}\right]= & \frac{1}{\ell_{N}} \mathbb{E}\left[\imath\left(X^{\ell_{N}} ; Y^{\ell_{N}}\right)\right] \\
= & \frac{1}{\ell_{N}} \mathbb{E}\left[\ln \frac{P_{Y^{\ell_{N}} \mid X^{\ell_{N}}}\left(Y^{\ell_{N}} \mid X^{\ell_{N}}\right)}{P_{\tilde{Y}^{\ell_{N}}}\left(Y^{\ell_{N}}\right)}\right] \\
& -\frac{1}{\ell_{N}} \mathbb{E}\left[\ln \frac{P_{Y^{\ell_{N}}}\left(Y^{\ell_{N}}\right)}{P_{\tilde{Y}^{\ell_{N}}}\left(Y^{\ell_{N}}\right)}\right] \\
\geq & C(P)-\frac{1}{\ell_{N}} \max _{y^{\ell_{N}} \in \mathbb{R}^{\ell_{N}}} \ln \frac{P_{Y^{\ell_{N}}}\left(y^{\ell_{N}}\right)}{P_{\tilde{Y} \ell_{N}}\left(y^{\ell_{N}}\right)} \\
\geq & C(P)-\frac{1}{\ell_{N}} \ln J(P),
\end{aligned}
$$

where $\tilde{Y}^{\ell_{N}}$ is a $\mathcal{N}\left(0,(1+P) \boldsymbol{|}_{\ell_{N}}\right)$ random vector, and $J(P)$ is given in (49). Inequalities (150) and (151) follow from (68) and Lemma 2, respectively. Similarly,

$$
\operatorname{Var}\left[B_{1}\right]=\frac{V(P)}{\ell_{N}}(1+o(1))
$$

for any $\ell_{N} \rightarrow \infty$ as $N \rightarrow \infty$. Applying Lemma 5 to (144), where $\left(X_{1}, X_{2}, \ldots\right)$ and $\gamma$ in Lemma 5 are set as $\left(B_{1}, B_{2}, \ldots\right)$ and $\frac{\gamma}{\ell_{N}}$, respectively, we get

$$
\begin{aligned}
\mathbb{E}[\xi] & \leq \frac{\frac{\gamma}{\ell_{N}}}{\mathbb{E}\left[B_{1}\right]}+\frac{\mathbb{E}\left[\left(\max \left\{0, B_{1}\right\}\right)^{2}\right]}{\left(\mathbb{E}\left[B_{1}\right]\right)^{2}} \\
& \leq \frac{\frac{\gamma}{\ell_{N}}}{C(P)-\frac{1}{\ell_{N}} \ln J(P)}+1+\frac{\operatorname{Var}\left[B_{1}\right]}{\left(\mathbb{E}\left[B_{1}\right]\right)^{2}},
\end{aligned}
$$

where (154) follows from (151) and the observation that $\mathbb{E}\left[\left(\max \left\{0, B_{1}\right\}\right)^{2}\right] \leq \mathbb{E}\left[B_{1}^{2}\right]$. Setting

$$
\gamma=N C(P)-\ell_{N} C(P)-\frac{N}{\ell_{N}} \ln J(P)+O(1),
$$

we get from (144) and (151)-(154),

$$
\mathbb{E}[\tau] \leq N .
$$

By (143), $\mathbb{E}\left[\tau^{*}\right]$ is also bounded by $N$.

To maximize the right-hand side of (155), we set

$$
\ell_{N} \triangleq \sqrt{N \frac{\ln J(P)}{C(P)}} .
$$

Error probability analysis: Following the steps in (39)-(47), the average error probability is bounded as

$$
\begin{aligned}
& \mathbb{P}\left[\mathrm{g}_{\tau^{*}}\left(U, Y^{n_{\tau^{*}}}\right) \neq W\right] \\
& \leq(M-1) \exp \{-\gamma\}+\mathbb{P}\left[\bigcup_{i=1}^{K}\left\{\left\|X^{n_{i}}\right\|^{2}>n_{i} P\right\}\right] .
\end{aligned}
$$

Note that, as argued in [6, eq. (109], $\mathbb{P}[\tau=\infty]=0$ by (156). Since the random codewords (57) satisfy the maximal power constraint (8) with probability 1, the second term in (158) is 0 . Setting

$$
\ln M=\gamma-\ln N
$$

bounds the error probability by $\frac{1}{N}$.

Combining (155), (157), and (159) completes the proof of Lemma 4.

2) Completing the proof of Theorem 4: The strategy is similar to that of the proof of Theorem 1 with the difference that we set

$$
\epsilon_{N}^{\prime} \triangleq \frac{1}{N^{\prime}}
$$

Before the transmission starts, we generate a random variable $D \sim \operatorname{Bernoulli}(p)$ with

$$
p \triangleq \frac{\epsilon-\epsilon_{N}^{\prime}}{1-\epsilon_{N}^{\prime}} .
$$

If $D=1$, then the decoder decodes to an arbitrary message at time $n_{1}=0$; otherwise the encoder and decoder use an $\left(N^{\prime},\left\{n_{k}\right\}_{k=2}^{\infty}, M, \epsilon_{N}^{\prime}, P\right)$ VLSF code. Following steps identical to (125)-(127), we see that the average error probability of the code is bounded by $\epsilon$, and that the average decoding time is $N=N^{\prime}(1-p)$, or

$$
N^{\prime}=\frac{N}{1-\epsilon}\left(1+O\left(\frac{1}{N}\right)\right) .
$$

Combining Lemma 4 and (162) completes the proof.

\section{REFERENCES}

[1] C. Shannon, "The zero error capacity of a noisy channel," IRE Trans. on Inf. Theory, vol. 2, no. 3, pp. 8-19, Sep. 1956.

[2] M. Horstein, "Sequential transmission using noiseless feedback," IEEE Trans. Inf. Theory, vol. 9, no. 3, pp. 136-143, Jul. 1963.

[3] J. Schalkwijk and T. Kailath, "A coding scheme for additive noise channels with feedback-I: No bandwidth constraint," IEEE Trans. Inf. Theory, vol. 12, no. 2, pp. 172-182, Apr. 1966.

[4] A. B. Wagner, N. V. Shende, and Y. Altuğ, "A new method for employing feedback to improve coding performance," IEEE Trans. Inf. Theory, vol. 66, no. 11, pp. 6660-6681, Nov. 2020.

[5] M. V. Burnashev, "Data transmission over a discrete channel with feedback: Random transmission time," Problems of Information Transmission, vol. 12, no. 4, pp. 10-30, 1976. 
[6] Y. Polyanskiy, H. V. Poor, and S. Verdú, "Feedback in the nonasymptotic regime," IEEE Trans. Inf. Theory, vol. 57, no. 8, pp. 49034925, Aug. 2011.

[7] Y. Polyanskiy, H. V. Poor, and S. Verdu, "Channel coding rate in the finite blocklength regime," IEEE Trans. Inf. Theory, vol. 56, no. 5, pp. 2307-2359, May 2010.

[8] S. L. Fong and V. Y. F. Tan, "Asymptotic expansions for the AWGN channel with feedback under a peak power constraint," in Proc. IEEE Int. Symp. Inf. Theory (ISIT), Hong Kong, China, Jun. 2015, pp. 311315.

[9] S. H. Kim, D. K. Sung, and T. Le-Ngoc, "Variable-length feedback codes under a strict delay constraint," IEEE Communications Letters, vol. 19, no. 4, pp. 513-516, Apr. 2015.

[10] Y. Altuğ, H. V. Poor, and S. Verdú, "Variable-length channel codes with probabilistic delay guarantees," in 53rd Annual Allerton Conference on Communication, Control, and Computing (Allerton), Monticello, IL, USA, Sep. 2015, pp. 642-649.

[11] L. V. Truong and V. Y. F. Tan, "On Gaussian MACs with variable-length feedback and non-vanishing error probabilities," arXiv:1609.00594v2, Sep. 2016

[12] — "On Gaussian MACs with variable-length feedback and nonvanishing error probabilities," IEEE Trans. Inf. Theor, vol. 64, no. 4, p. 2333-2346, Apr. 2018.

[13] K. F. Trillingsgaard, W. Yang, G. Durisi, and P. Popovski, "Commonmessage broadcast channels with feedback in the nonasymptotic regime: Stop feedback," IEEE Trans. Inf. Theory, vol. 64, no. 12, pp. 7686-7718, Dec. 2018.

[14] A. R. Williamson, T. Chen, and R. D. Wesel, "Variable-length convolutional coding for short blocklengths with decision feedback," IEEE Trans. Commun., vol. 63, no. 7, pp. 2389-2403, Jul. 2015.

[15] A. Heidarzadeh, J. Chamberland, R. D. Wesel, and P. Parag, "A systematic approach to incremental redundancy with application to erasure channels," IEEE Trans. Commun., vol. 67, no. 4, pp. 2620-2631, Apr. 2019.

[16] L. V. Truong, S. L. Fong, and V. Y. F. Tan, "On Gaussian channels with feedback under expected power constraints and with non-vanishing error probabilities," IEEE Trans. Inf. Theory, vol. 63, no. 3, pp. 1746-1765, Mar. 2017.

[17] V. Y. F. Tan and M. Tomamichel, "The third-order term in the normal approximation for the AWGN channel," IEEE Trans. Inf. Theory, vol. 61, no. 5, pp. 2430-2438, May 2015.

[18] W. Yang, G. Caire, G. Durisi, and Y. Polyanskiy, "Optimum power control at finite blocklength," IEEE Trans. Inf. Theory, vol. 61, no. 9, pp. 4598-4615, Sep. 2015.

[19] R. C. Yavas, V. Kostina, and M. Effros, "Random access channel coding in the finite blocklength regime," IEEE Trans. Inf. Theory, Dec. 2020, early access. doi: 10.1109/TIT.2020.3047630.

[20] Y. Polyanskiy and S. Verdú, "Channel dispersion and moderate deviations limits for memoryless channels," in 2010 48th Annual Allerton Conference on Communication, Control, and Computing (Allerton), 2010, pp. 1334-1339.

[21] V. V. Petrov, Sums of independent random variables. New York, USA: Springer, Berlin, Heidelberg, 1975.

[22] E. MolavianJazi and J. N. Laneman, "A second-order achievable rate region for Gaussian multi-access channels via a central limit theorem for functions," IEEE Trans. Inf. Theory, vol. 61, no. 12, pp. 6719-6733, Dec. 2015.

[23] R. C. Yavas, V. Kostina, and M. Effros, "Gaussian multiple and random access in the finite blocklength regime," in Proc. IEEE Int. Symp. Inf. Theory (ISIT), Los Angeles, CA, USA, June 2020, pp. 3013-3018.

[24] C. E. Shannon, "Probability of error for optimal codes in a Gaussian channel," The Bell System Technical Journal, vol. 38, no. 3, pp. 611-656, May 1959.

[25] E. MolavianJazi, "A unified approach to Gaussian channels with finite blocklength," Ph.D. dissertation, University of Notre Dame, Jul. 2014.

[26] G. Lorden, "On excess over the boundary," Ann. Math. Statist., vol. 41, no. 2, pp. 520-527, 041970. 